\title{
IMPACT OF REDUCTION OF RADIOMETRIC RESOLUTION IN HYPERSPECTRAL IMAGES ACQUIRED OVER FOREST FIELD
}

\author{
G. T. Miyoshi ${ }^{1,1}$, N. N. Imai ${ }^{1,2}$, A. M. G. Tommaselli ${ }^{1,2}$, E. Honkavaara ${ }^{3}$ \\ ${ }^{1}$ Post Graduate Program in Cartographic Science, São Paulo State University (UNESP), Presidente Prudente-SP, Brazil - \\ takahashi.gabi@gmail.com \\ ${ }^{2}$ Dept. of Cartography, São Paulo State University (UNESP), Presidente Prudente-SP, Brazil - (nilton.imai, a.tommaselli)@unesp.br \\ ${ }^{3}$ Finnish Geospatial Research Institute FGI, Geodeetinrinne 2, P.O. Box 15, FI-02431 Masala, Finland - eija.honkavaara@nls.fi
}

\section{Commission I, WG I/1}

KEY WORDS: Radiometric resolution, Hyperspectral image, Normalized Root Mean Square, Mean Square Percentage Error, Boxplot

\begin{abstract}
:
The objective of this study was to evaluate the impact of reducing the radiometric information of hyperspectral images. The image data was collected originally with 32 bits and rescaled to 8 and 16 bit/pixel. The images were acquired with a Rikola Hyperspectral Camera attached to an Unmanned Aerial Vehicle (UAV). After the geometric and radiometric processing of the images, a mosaic was obtained with pixels representing reflectance factor coded in 32 bits. Using the minimum and maximum values of each spectral band, a linear equation was thus applied to reduce the radiometric resolution of the original mosaic, from 32 bits to 8 bits and from 32 bits to 16 bits. Following, the Normalized Root Mean Square Error (NRMSE\%) and the Mean Absolute Percentage Error (MAPE\%) were used to evaluate the results, showing that for the 8 bits mosaic, the loss of information was higher. For this radiometric resolution rescaling, the MAPE\% achieved values until $22.486 \%$ and the highest NRMSE\% value was $0.455 \%$ while, for the 16 bits mosaics, the highest MAPE\% and NRMSE $\%$ values were $0.069 \%$ and $0.002 \%$, respectively. Finally, it can be inferred that the impact of radiometric transformation can be considered as negligible for the hyperspectral mosaic with 16 bits of radiometric resolution, which presented lower values of NRMSE \% and MAE \% and could not affect the mosaic analysis.
\end{abstract}

\section{INTRODUCTION}

The use of Unmanned Aerial Vehicle (UAV) remote sensing is expected to revolutionize various environmental applications due to their capability to capture datasets at desired spatial, spectral, radiometric and temporal resolutions (Anderson and Gaston, 2013; Sanchez-Azofeifa et al., 2017). UAV based spectrometry is one of the rapidly developing fields (Adão et al., 2017; Aasen et al., 2018).

The radiometric resolution of a sensor is an important characteristic to be explored since it reflects the quantization level that the sensor is capable to record and represent the reflected energy of targets, besides influencing the analysis of targets spectral features (Orych et al., 2014; Tucker, 1980).

Although this subject is not commonly studied, it is of wide interest for the research community and practical applications, especially when hyperspectral images with high spatial resolution are used. This kind of data could be noisier than images with a lower radiometric resolution, affecting results such as the obtained through image classification, and their processing can be very time consuming, harming the product generation. In addition, usually there is a need for huge data storage and processing for this kind of data. Therefore, some level of radiometric resolution reduction should be interesting.

For forestry applications it is interesting to evaluate what should be the most suitable number of bits necessary to store data reliably. Furthermore, this study can be of interest to improve the product generation of hyperspectral images acquired with the increased use of Unmanned Aerial Vehicles, which can provide rapid image data sets (Oliveira et al., 2018).

In this sense, the objective of this study is to evaluate the impact of reducing the radiometric information of hyperspectral images comparing the data with 32 bits of a mosaic composed by hyperspectral images with those obtained by rescaling to 8 and 16 bits.

\section{METHODOLOGY}

\subsection{Image acquisition - Hyperspectral camera and UAV}

To acquire the hyperspectral images, the Rikola Hyperspectral Camera, model DT-0011, was used. This camera is commercialized by Senop Ltd. and is based on the tuneable Fabry-Pérot Interferometer (FPI) which makes the acquisition of different spectral bands possible. The approximated focal length of the sensor is $8.7 \mathrm{~mm}$ and the frame format sensors are sized of $1017 \times 648$ pixels with a pixel size of $5.5 \mu \mathrm{m}$. The different airgaps of the FPI can be settled in order to acquire spectral images ranging from $500 \mathrm{~nm}$ to $900 \mathrm{~nm}$. In total, 25 spectral bands were set according the spectral response of targets present in the neighbourhood of the imagined area (Miyoshi et al., 2018). Table 1 shows the spectral setting of the camera with the respective Full Width at Half Maximum (FWHM) in nanometres (nm).

\footnotetext{
* Corresponding author
} 
Central wavelengths of each spectral band (nm): 506.22, 519.94, $535.09,550.39,565.10,580.16,591.90,609.00,620.22,628.73$ $650.96,659.72,669.75,679.84,690.28,700.28,710.06,720.17$ $729.57,740.42,750.16,769.89,780.49,790.30,819.66$

FWHM (nm): $12.44,17.38,16.84,16.53,17.26,15.95,16.61$, $15.08,16.26,15.30,14.44,16.83,19.80,20.45,18.87,18.94,19.70$, $19.31,19.01,17.98,17.97,18.72,17.36,17.39,17.84$

Table 1. Spectral settings of the hyperspectral camera

The camera was mounted in a quadcopter UAV, model UX4. This UAV was developed specially to be used with the Rikola Hyperspectral Camera and it system is composed by the by PixHawk autopilot, a GoPro Hero 4 Black camera and a GNSS receiver. As energy source for the UAV system and it sensors two 6-cell batteries of 22 volts each and one smaller batterie of 3 -cell and 11 volts are used. Figure 1 shows the UX4 UAV and the Rikola Hyperspectral Camera during a flight campaign.

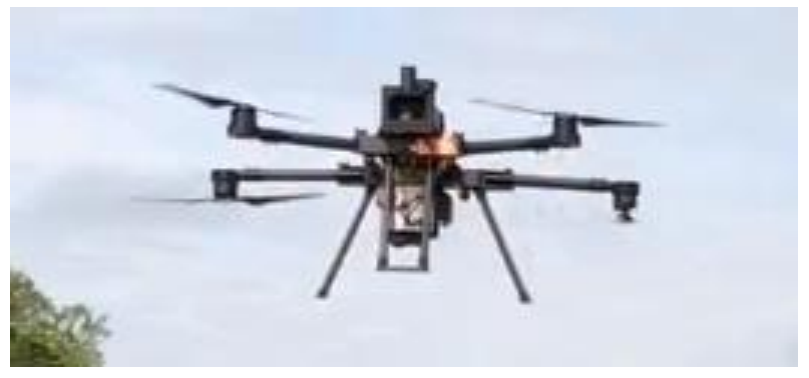

Figure 1. UX4 UAV and its payload

The image data set were acquired in July, $1^{\text {st }}$ of 2017 , between $10 \mathrm{~h} 14$ and $10 \mathrm{~h} 24$, local time (UTC-3). The flight height was $160 \mathrm{~m}$ above the ground, providing images with Ground Sample Distance (GSD) of approximately $10 \mathrm{~cm}$, excluding the canopy height. The flight speed was set to $4 \mathrm{~m} / \mathrm{s}$ and the time interval between cubes acquisitions was $2 \mathrm{~s}$, providing more than $70 \%$ of forward overlap.

A fragment of the Ponte Branca reservoir was used as study area. This area belongs to the Black Lion Tamarin Ecological Station, managed by Chico Mendes Institute of Biodiversity. The area comprises a small road and forest with young and mature species. The approximated area is 3.95 ha comprising more than 20 tree species (Berveglieri el al., 2016). Figure 2 shows the Ponte Branca area, in the western part of São Paulo State and highlighted in red in the right part of the figure.

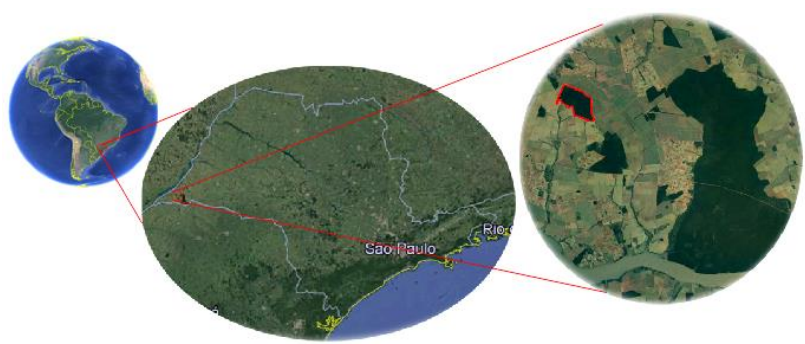

Figure 2. Location of the study area. Ponte Branca is highlighted in red in the right part of the figure.

\subsection{Imaging processing}

The images were originally acquired with a radiometric resolution of $12 \mathrm{bit} /$ pixel, being rescaled to 32 bits in the first step of the imaging processing (Rikola, 2015). This first step applies the dark current and smile correction and the radiometric correction of each image. The Hyperspectral Imager software provided by the manufacture is used for this purpose and relies on a dark image acquired before the images acquisition and calibration factors obtained in laboratory. As result, the Digital Number (DN) of each image pixel is transformed to radiance values in $\left[\mathrm{mW} /\left(\mathrm{m}^{2} . \mathrm{sr} . \mathrm{nm}\right)\right]$.

In order to obtain the interior orientation parameters (IOP) of each sensor and the exterior orientation parameters (EOP) of each image and spectral band, the geometric processing is applied. This step is performed with AgiSoft Photoscan software, which is based on Structure from Motion algorithms to find common points among the images and produce point clouds and Digital Surface Models (DSMs) (Nevalainen et al., 2017).

As input for this step, four spectral bands of each image were used. The reason to use those bands and not only one is the slightly difference in the spatial position of each band, caused by the FPI principle to acquire the images for different wavelengths. The remaining bands are not used to reduce the computational load. In the following step the procedure developed by Honkavaara et al. (2017) was used to determine the EOP for the other spectral bands. In this sense, the bands centred in $535.09 \mathrm{~nm}, 609.00 \mathrm{~nm}, 679.84 \mathrm{~nm}$ and $769.89 \mathrm{~nm}$ were chosen. They were selected considering the spectral bands in each sensor and the time to acquire those 25 bands. For $5 \mathrm{~ms}$ of integration time and for 25 spectral bands, is necessary $0.8 \mathrm{~s}$ to acquire each hypercube.

The images were used in the same process, that is, simultaneously, to refine the IOP using the self-calibrating adjustment and to acquire refined EOP values for the four spectral bands. Moreover, point clouds of the area and a DSM were produced to be used in the radiometric block adjustment and ortomosaic generation.

The technique of radiometric block adjustment of the hyperspectral images was developed by Honkavaara et al. (2013), and it considers the illumination changes of each image, which can be caused by different geometries of image acquisition and by anisotropy of targets. It uses a Bidirectional Reflectance Distribution Function model developed by Walthall et al. (1985) and homologous points in the images to perform the correction. In addition, the software of Honkavaara et al. (2013) uses point clouds and a DSM generated in the previous processing in AgiSoft Photoscan.

Finally, the empirical line method (Smith and Milton, 1999) is applied to transform the pixels values to reflectance factor values. Three radiometric reference targets with average reflectance factor of $4 \%, 11 \%$ and $37 \%$ were correlated with the pixel values of each spectral band in order to calculate the linear transformation. Thus, the final product was a geometric and radiometric corrected mosaic of the hyperspectral images with pixels representing reflectance factor values in 32 bits of radiometric resolution.

\subsection{Different radiometric resolutions}

The first step to verify the impact of reducing the radiometric resolution was the selection of a sample of the total area. This sample comprises an area of approximately $200 \mathrm{~m}$ by $100 \mathrm{~m}$ ( 2 ha) and was chosen to represent the full area and to reduce 
the computational load. Following, the minimum and maximum values of each spectral band were determined using a routine developed in Python.

The minimum and maximum values of each spectral band were used in a linear equation to reduce the radiometric resolution of the mosaic, i.e., to rescale the mosaic from 32 bits to 8 bits and from 32 bits to 16 bits. Considering the mosaic of 8 bits, the minimum value of the original mosaic was set to zero value and the maximum to 255 value. This is the well known MinMax normalization technique used for image contrast expansion. The same relationship was applied to the 16 bits mosaic using zero and the 65,535 values. Equation 1 summarizes the relationship used to rescale the mosaic.

$$
\frac{\max _{32 \text { bits }}-x}{x-\min _{32 \text { bits }}}=\frac{\max _{n e w}-y}{y-\min _{n e w}}
$$

where $\max _{32 b i t s}=$ maximum value of the pixel for the original mosaic (with 32 bits of radiometric resolution) $\min _{32 b i t s}=$ minimum value of the pixel for the original mosaic (with 32 bits of radiometric resolution) $x=$ value of each pixel of the original mosaic max $_{\text {new }}=$ maximum value of the pixel for the rescaled mosaic

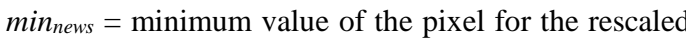
mosaic

$y=$ new value to be calculated to rescale the mosaic to a new radiometric resolution

This equation was applied to each spectral band to transform the original mosaic in two new mosaics, decreasing the radiometric resolution from 32 bits to 8 bits of radiometric resolution and from 32 bits to 16 bits.

Following the methodology, a new mosaic rescaling was performed, aiming to change the radiometric resolution from 8 to 32 bits and from 16 to 32 bits. By manipulating Equation 1, the new values were computed. It can be noticed that this step was required in order to compare the difference between the original mosaic and those originated from the 8 and 16 bits radiometric resolution mosaics.

The metrics applied to verify the differences were the Normalized Root Mean Square Error (NRMSE\%) and the Mean Absolute Percentage Error (MAPE\%) (Mayer and Butler, 1993; Willmott and Matsuura, 2005; Watanabe et al., 2016). Those metrics were calculated using 15 check points, where 3 of the points were placed in the radiometric reference targets and the other 12 were randomly distributed in the study area (Figure 3 ). In addition, the boxplot of each spectral band, for each rescaling, were generated in order to verify the distribution of the data and the number of outliers.

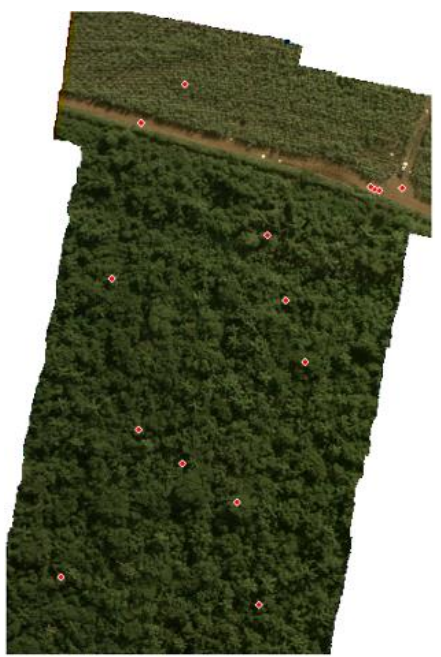

Figure 3. Study area and check points used to evaluate the different radiometric resolutions

\section{RESULTS}

Tables 2 and 3 present the NRMSE\% and the MAPE\% for the rescaled mosaic from 32 bits to 8 bits. Analysing Table 3, it can be noticed that the NRMSE\% varied from $0.130 \%$ to $0.455 \%$, while the minimum MAPE\% was $0.540 \%$ and the maximum value was $22.486 \%$. These results showed different error magnitudes, which may also affect the MAPE\% values. Looking the check points, it was noticed that the biggest error occurred in one point located in a shadowed area, which may also affect the results for the 8 bis rescaled mosaic.

\begin{tabular}{|cc|cc|cc|}
\hline Band & NRSME \% & Band & NRSME \% & Band & NRSME \% \\
\hline 1 & 0.134 & 10 & 0.176 & 18 & 0.304 \\
2 & 0.130 & 11 & 0.445 & 19 & 0.316 \\
3 & 0.136 & 12 & 0.362 & 20 & 0.343 \\
4 & 0.182 & 13 & 0.304 & 21 & 0.450 \\
5 & 0.330 & 14 & 0.271 & 22 & 0.324 \\
6 & 0.257 & 15 & 0.268 & 23 & 0.455 \\
7 & 0.275 & 16 & 0.277 & 24 & 0.354 \\
8 & 0.343 & 17 & 0.275 & 25 & 0.305 \\
9 & 0.363 & & & & \\
\hline
\end{tabular}

Table 2. NRMSE\% obtained from the radiometric resolution reduction to 8 bits

\begin{tabular}{|cc|cc|cc|}
\hline Band & MAPE \% & Band & MAPE \% & Band & MAPE \% \\
\hline 1 & 1.155 & 10 & 4.269 & 18 & 1.546 \\
2 & 1.774 & 11 & 5.253 & 19 & 0.755 \\
3 & 1.321 & 12 & 5.183 & 20 & 0.720 \\
4 & 1.125 & 13 & 22.486 & 21 & 1.093 \\
5 & 2.838 & 14 & 2.856 & 22 & 0.579 \\
6 & 2.744 & 15 & 6.904 & 23 & 1.122 \\
7 & 5.642 & 16 & 8.238 & 24 & 0.671 \\
8 & 5.130 & 17 & 2.339 & 25 & 0.540 \\
9 & 18.494 & & & & \\
\hline
\end{tabular}

Table 3. MAPE\% obtained from the radiometric resolution reduction to 8 bits 
Considering the second radiometric resolution reduction, from 32 bits to 16 bits, the NRSM\% and MAPE\% were also calculated using 15 points of the mosaic. The results are shown in Tables 4 and 5 , where is possible to see that the average NRMSE\% is lower than $0.002 \%$, with maximum value of $1.78 \mathrm{E}-03$. This result shows a high degree of similarity between the original mosaic and the rescaled one, also proven by the MAPE\% values, which varies from $0.002 \%$ to $0.069 \%$.

\begin{tabular}{|cc|cc|cc|}
\hline Band & NRSME \% & Band & NRSME \% & Band & NRSME \% \\
\hline 1 & $7.89 \mathrm{E}-04$ & 10 & $6.41 \mathrm{E}-04$ & 18 & $1.22 \mathrm{E}-03$ \\
2 & $6.31 \mathrm{E}-04$ & 11 & $1.74 \mathrm{E}-03$ & 19 & $1.51 \mathrm{E}-03$ \\
3 & $5.85 \mathrm{E}-04$ & 12 & $1.03 \mathrm{E}-03$ & 20 & $1.59 \mathrm{E}-03$ \\
4 & $6.59 \mathrm{E}-04$ & 13 & $1.10 \mathrm{E}-03$ & 21 & $1.78 \mathrm{E}-03$ \\
5 & $1.25 \mathrm{E}-03$ & 14 & $1.16 \mathrm{E}-03$ & 22 & $1.32 \mathrm{E}-03$ \\
6 & $1.36 \mathrm{E}-03$ & 15 & $1.01 \mathrm{E}-03$ & 23 & $1.33 \mathrm{E}-03$ \\
7 & $1.58 \mathrm{E}-03$ & 16 & $9.96 \mathrm{E}-04$ & 24 & $1.59 \mathrm{E}-03$ \\
8 & $1.31 \mathrm{E}-03$ & 17 & $1.12 \mathrm{E}-03$ & 25 & $1.34 \mathrm{E}-03$ \\
9 & $1.33 \mathrm{E}-03$ & & & & \\
\hline
\end{tabular}

Table 4. NRMSE\% obtained from the radiometric resolution reduction to 16 bits

\begin{tabular}{|cc|cc|cc|}
\hline Band & MAPE \% & Band & MAPE \% & Band & MAPE \% \\
\hline 1 & 0.008 & 10 & 0.011 & 18 & 0.004 \\
2 & 0.007 & 11 & 0.025 & 19 & 0.005 \\
3 & 0.007 & 12 & 0.013 & 20 & 0.003 \\
4 & 0.006 & 13 & 0.069 & 21 & 0.003 \\
5 & 0.013 & 14 & 0.026 & 22 & 0.002 \\
6 & 0.020 & 15 & 0.029 & 23 & 0.003 \\
7 & 0.021 & 16 & 0.023 & 24 & 0.004 \\
8 & 0.015 & 17 & 0.006 & 25 & 0.003 \\
9 & 0.024 & & & & 0.004 \\
\hline
\end{tabular}

Table 5. MAPE\% obtained from the radiometric resolution reduction to 16 bits

The results obtained with the boxplots showed that the variability of the tree mosaics was similar; however, the one rescaled to 8 bits was the one with less outliers, being probably the one with less noise, followed by the 16 bits rescaled mosaic and by the original one.

\section{CONCLUSION}

Considering the results and the aim of this work, it can be inferred that the impact of radiometric transformation can be considered as negligible for the hyperspectral images with 16 bits of radiometric resolution, which presented lower values of NRMSE \% and MAPE\%. The results obtained for the 8 bits mosaic showed less noise, however higher errors. Those values indicate that for this case, the reduction of the radiometric resolution could affect further analysis such as the image classification, since the spectral response of targets could be changed. Reduced radiometric resolution with the 8 bit images can also impact digital surface model generation based on image matching.

\section{ACKNOWLEDGEMENTS}

This research has been jointly funded by the São Paulo Research Foundation (FAPESP - grant 2013/50426-4) and Academy of Finland - grant number 305994), as well the Conselho Nacional de Desenvolvimento Científico e Tecnológico (CNPq) (grant 153854/2016-2) and Coordenação de Aperfeiçoamento de Pessoal de Nível Superior (CAPES) through a PhD scholarship.

\section{REFERENCES}

Aasen, H.;, Honkavaara, E.; Lucieer, A.; and Zarco-Tejada, P.J., 2018. Quantitative Remote Sensing at Ultra-High Resolution with UAV Spectroscopy: A Review of Sensor Technology, Measurement Procedures, and Data Correction Workflows. Remote Sens.ing 2018, 10, 1091.

Anderson, K. and Gaston, K.J., 2013. Lightweight unmanned aerial vehicles will revolutionize spatial ecology. Frontiers in Ecology and the Environment, 11(3), pp. 138-146.

Adão, T., Hruška, J., Pádua, L., Bessa, J., Peres, E., Morais, R., and Sousa, J., 2017. Hyperspectral Imaging: A Review on UAV-Based Sensors, Data Processing and Applications for Agriculture and Forestry. Remote Sensing. 2017, 9(11), 1110.

Berveglieri, A., Tommaselli, A. M. G., Imai, N. N., Ribeiro, E. A. W., Guimarães, R. B., and Honkavaara, E., 2016. Identification of Successional Stages and Cover Changes of Tropical Forest Based on Digital Surface Model Analysis. IEEE Journal of Selected Topics in Applied Earth Observations and Remote Sensing, 9(12), pp. 5385-5397.

Honkavaara, E., Rosnell, T., Oliveira, R., and Tommaselli, A. M. G. T., 2017. A. Bband registration of tuneable frame format hyperspectral UAV imagers in complex scenes. ISPRS Journal of Photogrammetry and Remote Sensing 2017, 134, pp. 96-109.

Honkavaara, E., Saari, H., Kaivosoja, J., Pölönen, I., Hakala, T., Litkey, P., Mäkynen, J. and Pesonen, L., 2013. Processing and assessment of spectrometric, stereoscopic imagery collected using a lightweight UAV spectral camera for precision agriculture. Remote Sensing, 5(10), pp. 5006-5039.

Mayer, D. G., and Butler, D. G., 1993. Statistical validation. Ecological modelling, 68(1-2), 21-32.

Miyoshi, G. T., Imai, N. N., Tommaselli, A. M. G., Honkavaara, E., Näsi, R., and Moriya, É. A. S., 2018. Radiometric block adjustment of hyperspectral image blocks in the Brazilian environment. International Journal of Remote Sensing, 1-21.

Nevalainen, O., Honkavaara, E., Tuominen, S., Viljanen, N., Hakala, T., Yu, X., Hyyppä, J., Saari, H., Pölönen, I., Imai, N. N., and Tommaselli, A. M. G., 2017. et al. Individual Tree Detection and Classification with UAV-Based Photogrammetric Point Clouds and Hyperspectral Imaging. Remote Sensing, 9, 185.

Oliveira, R. A., Khoramshahi, E., Suomalainen, J., Hakala, T., Viljanen, N., and Honkavaara, E., 2018. Real-time and postprocessed georeferencing for hyperpspectral drone Remote Sensing. In: International Archives of the Photogrammetry, 
Remote Sensing and Spatial Information Sciences, 2018 ISPRS Congress, pp. 789-795.

Orych, A., Walczykowski, P., Jenerowicz, A., and Zdunek, Z.,2014. Impact of the cameras radiometric resolution on the accuracy of determining spectral reflectance coefficients. In: International Archives of the Photogrammetry, Remote Sensing and Spatial Information Sciences, 2014 ISPRS Congress, pp. 347-349.

Rikola Ltd., 2015. Rikola Hyperspectral Imager - Quick start Guide. Kaitoväylä, Oulu, Finland.

Sanchez-Azofeifa, A., Antonio Guzmán, J., Campos, C. A., Castro, S., Garcia-Millan, V., Nightingale, J., and Rankine, C., 2017. Twenty-first century remote sensing technologies are revolutionizing the study of tropical forests. Biotropica, 49, pp. 604-619.

Smith, G and Milton E, 1999. The use of the empirical line method to calibrate remotely sensed data to reflectance. International Journal of Remote Sensing, 20(13), pp. 26532662.

Tucker, C. J., 1980. Radiometric resolution for monitoring vegetation How many bits are needed?. International Journal of Remote Sensing, 1(3), 241-254.

Walthall, C. L., Norman, J. M., Welles, J. M., Campbell, G., and Blad, B. L. Simple equation to approximate the bidirectional reflectance from vegetative canopies and bare soil surfaces. Applied Optics, v. 24, n. (3), pp. 383-387, 1985.

Watanabe, F., Mishra, D. R., Astuti, I., Rodrigues, T., Alcântara, E., Imai, N. N., and Barbosa, C., 2016. Parametrization and calibration of a quasi-analytical algorithm for tropical eutrophic waters. ISPRS Journal of Photogrammetry and Remote Sensing, 121, 28-47. 